\title{
Adenoid cystic carcinoma of trachea masquerading as papillary thyroid cancer - a case report
}

\author{
Abhay K. Kattepur, Darshan Patil, Srinivas K.G, Shivananda Swamy, Vijayashree Murthy, \\ Amarendra S., K S. Gopinath \\ Department of Surgical Oncology, HCG-Bangalore Institute of Oncology, Bangalore, India \\ Correspondence: Darshan Patil. Address: Department of Surgical Oncology, HCG-Bangalore Institute of Oncology, \\ \#44-45/2, 2nd Cross, RRMR Extension (Off Lalbagh Double Road) Bangalore - 560027, India. E-mail: \\ darshupatil28@gmail.com
}

Received: March 3, 2014

Accepted: April 15, 2014

Online Published: April 28, 2014

DOI : $10.5430 /$ crcp.v1n2p53

URL: http://dx.doi.org/10.5430/crcp.v1n2p53

\section{Abstract}

Introduction: Thyroid nodules are common clinical entity. Though indolent in nature, they can be invasive too. However, tracheal tumours are relatively rare and come into clinical picture when patients show symptoms of tracheal obstruction.

Case report: We report a 45 year old female who presented with cytology proven papillary carcinoma thyroid with clinical and radiological evidence of tracheal involvement. Patient underwent total thyroidectomy along with en bloc segmental tracheal resection with end to end anastomosis. However the pathology revealed adenoid cystic carcinoma of trachea.

Conclusion: Adenoid cystic carcinomas of trachea are rare tumors and they can present as thyromegaly secondary to thyroid involvement. Surgeons should have a high index of suspicion for diagnosis. Surgical resection forms the mainstay of treatment.

\section{Key words}

Thyromegaly, Tracheal tumor, Adenoid cystic carcinoma

\section{I ntroduction}

The frequency of thyroid cancer in India among all the cancer cases is $0.1 \%-0.2 \%{ }^{[1]}$. Malignant thyroid swellings account for $8.2 \%$ of new cancer cases in India. On the contrary, tracheal tumours are relatively rare ${ }^{[2]}$ accounting for less than $0.2 \%$ of all malignancies of the respiratory tract ${ }^{[3]}$ and for under $0.1 \%$ of the cancer related deaths every year ${ }^{[4]}$. Direct invasion of the trachea by thyroid tumors is an unusual but well-documented clinical scenario. However the converse, that is, a tracheal tumour invading the thyroid and presenting as a thyromegaly is rare ${ }^{[5]}$. We present the case of a 45 year old woman who presented with a compressive thyroid swelling. A comprehensive review of literature with similar clinical presentation, diagnostic dilemma and prognosis of patients with the rare Adenoid cystic carcinoma (ACC) of trachea are discussed. 


\section{Case details}

A 45 year old female presented with history of occasional breathlessness and hoarseness of voice of two months duration. She was evaluated and diagnosed to have a cytology proven papillary thyroid cancer by a referring hospital. Past medical history was not significant for any illness or surgery.

General physical examination revealed no abnormalities and no palpable lymphadenopathy. Neck examination revealed $4 \mathrm{~cm} \times 4.5 \mathrm{~cm}$ thyromegaly involving both lobes. Mild stridor was also noticed though the patient was comfortable. Ultrasound of neck revealed enlarged right lobe of thyroid with diffuse heterogeneity with retro-tracheal extension and a normal left lobe. A Computed tomography (CT) scan of neck demonstrated a large ill-defined hypodense lesion in the right lobe of thyroid with 3rd to 5th tracheal ring involvement (see Figures 1, 2 and 3). Fine needle aspiration cytology of the thyroid swelling demonstrated papillary carcinoma thyroid. Thus a diagnosis of papillary carcinoma thyroid with tracheal involvement was made and patient prepared for surgery.

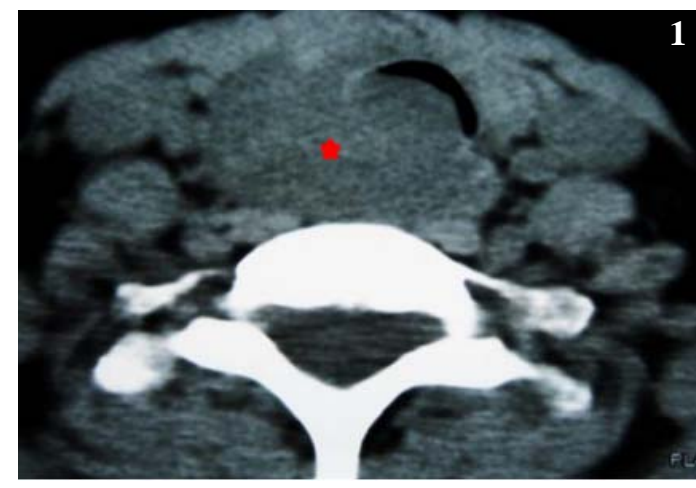

Figure 1. CT scan showing the tumour extent into the tracheal lumen

Figure 2. Coronal view with tumour in the tracheal lumen

Figure 3. Sagittal view of the tumour
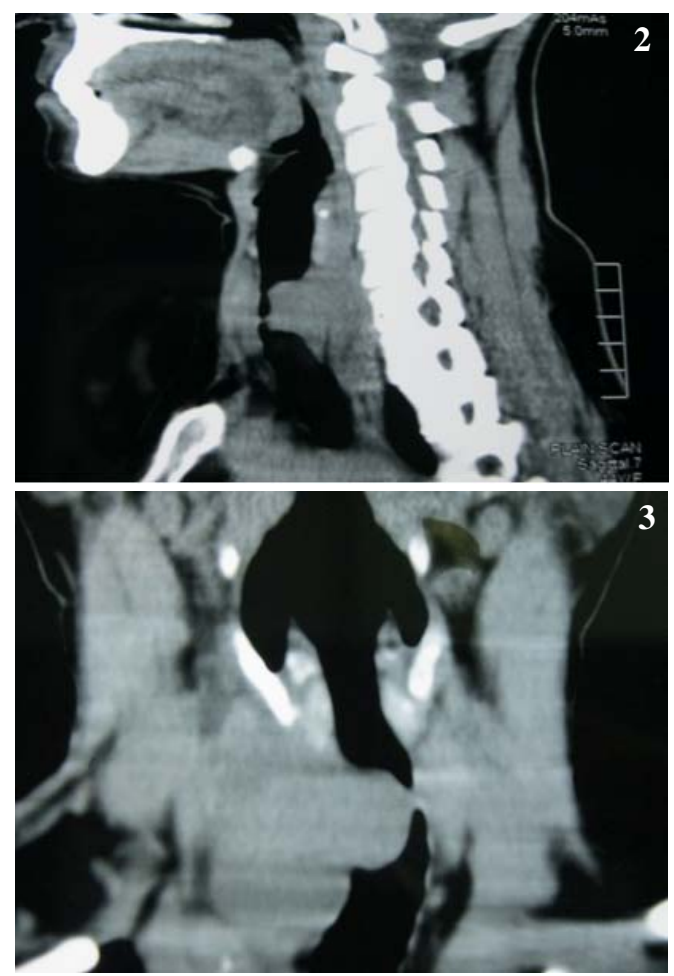

Pre-operative bronchoscopy revealed an intra-luminal nodule extending from the 3rd to 5th tracheal rings. Since the preoperative investigations revealed a malignant thyroid lesion with tracheal involvement, decision was to perform a standard total thyroidectomy with tracheal resection. The thyroid was approached using a standard neck incision. Intraoperative findings included hard nodularity of both lobes, right lobe measuring $2.5 \mathrm{~cm} \times 2 \mathrm{~cm}$ and left measuring $2 \mathrm{~cm}$ $\times 1.8 \mathrm{~cm}$ unicentric, with retrosternal extension. Total thyroidectomy was performed with identification and preservation of both recurrent laryngeal nerves and parathyroid glands. Central compartment clearance was done and through the same incision, the tissues in the superior mediastinum were cleared. The entire nodal tissues were brought to the centre before tracheal mobilization. Release of hyoid bone was done. Segmental tracheal resection with end to end anastomosis was performed (see Figures 4, 5). Following surgery, neck was kept flexed using a Guardian's stitch. Post operatively, patient was managed in the ICU with oxygen supplements and nebulisations.

Histopathology report revealed adenoid cystic carcinoma of the minor salivary gland of trachea measuring $2.2 \mathrm{~cm} \times$ $1.5 \mathrm{~cm} \times 1 \mathrm{~cm}$ involving the cartilage (see Figure 6) and thyroid gland with capsular, lymphovascular and perineural invasion. A parathyroid gland was also involved by the tumour. Thyroid also showed chronic lymphocytic thyroiditis. 
Microscopy revealed tumour cells arranged in cribriform pattern (see Figures 7 and 8). Immunohistochemistry showed only focal $(<5 \%)$ c-kit positivity.

Patient received adjuvant radiation and is found to be disease free after six months of follow up.

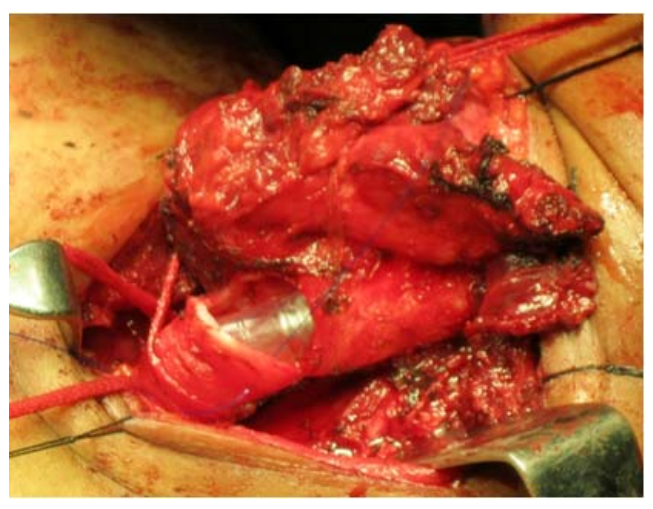

Figure 4. Tracheotomy done

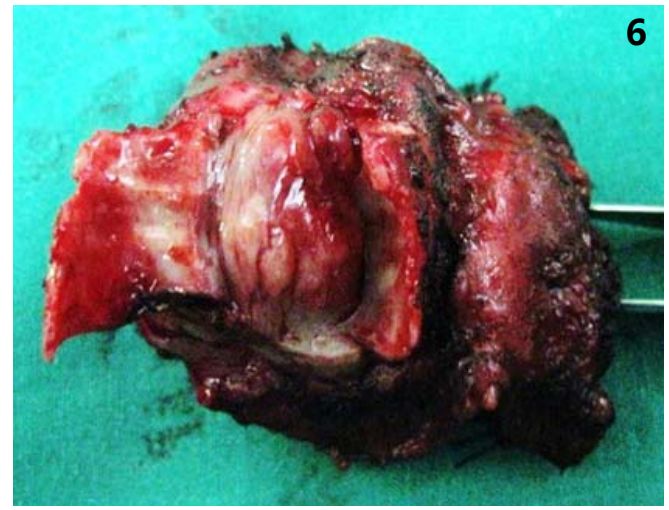

Figure 6. Cut specimen with tumour

Figure 7. Low power view $(10 \times)$ showing the basophilic stained tumour cells

Figure 8. Tumor cells in $40 \times$ high power view with a cribriform pattern of arrangement

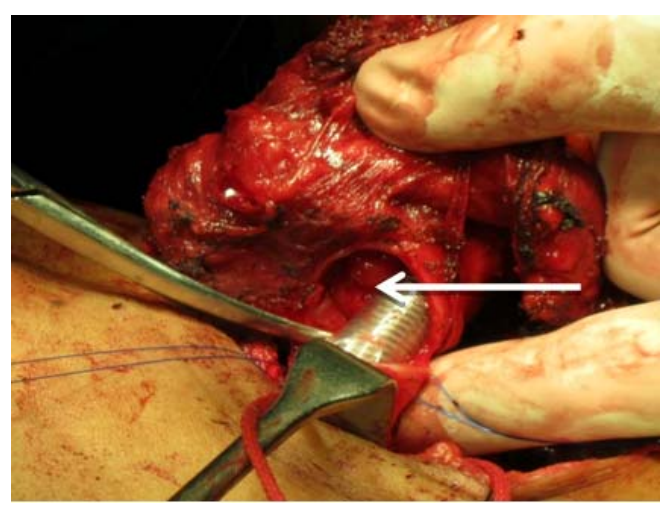

Figure 5. Tracheal lumen showing a growth

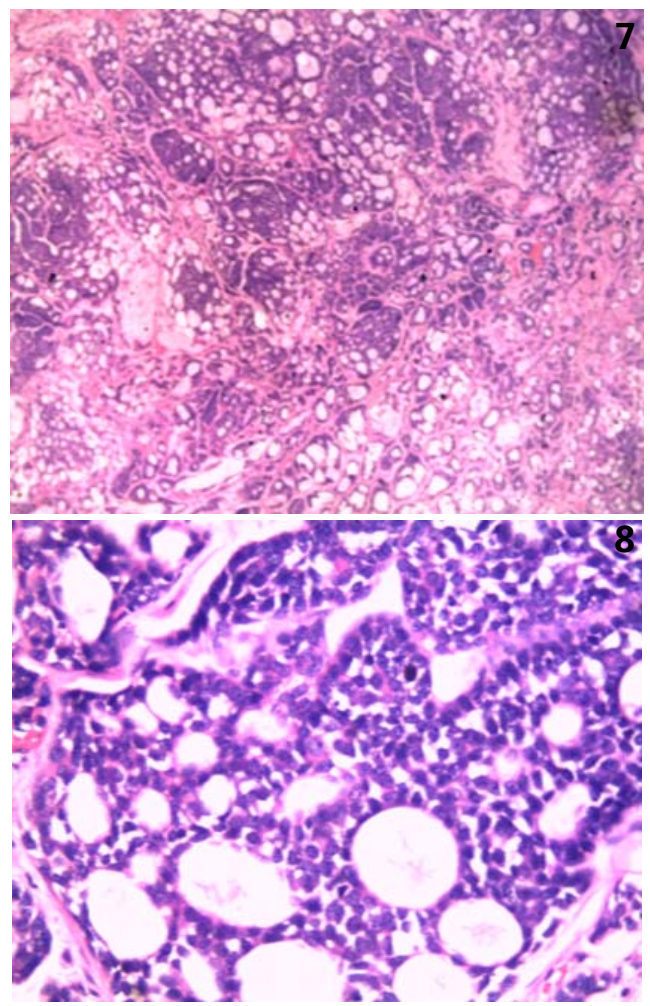

\section{Discussion}

After squamous cell carcinoma, ACC is the second most common tracheal malignancy ${ }^{[4]}$ accounting for about $10 \%-20 \%$ of all tracheal tumours ${ }^{[6]}$ and $80 \%$ of all tracheo-bronchial gland tumours ${ }^{[7]}$. The clinical and pathologic features of ACC of the trachea was first reported by Billroth in $1859^{[4]}$. They arise from the mixed sero-mucinous glands of the tracheo-bronchial submucosa. They are the most common salivary gland variety of neoplasms found in the lung ${ }^{[7]}$. These tumours commonly occur in the trachea or main stem bronchi. They can be located at any part of the trachea with preponderance to the upper part. In the present case, the lower part of trachea was involved. 
ACCs grossly present as sessile, polypoidal, annular or diffusely infiltrative nodules with heaped-up margins ${ }^{[2]}$. Histologically, they are identical to ACCs of the salivary glands often demonstrating a tendency toward submucosal extension. On microscopy, ACCs show monomorphic population of small basaloid cells with high nucleo-cytoplasmic ratio, coarse chromatin and small nucleoli arranged in cell nests ${ }^{[5]}$. The cells in these nests are separated by well-defined cystic spaces containing a mucinous substance that stains strongly with alcian blue and weakly with Periodic Acid - Schiff (PAS) ${ }^{[8]}$. The table enumerates the differences between ACC and papillary thyroid cancer (PTC), the commonest thyroid cancer on microscopic examination. The presence of acellular hyaline stroma in globular or cylindromatous formations is often seen in the cribriform and tubular subtypes of ACC. However, to diagnose adenoid cystic carcinoma solely based on these globules often results in an erroneous diagnosis ${ }^{[5]}$. The hallmark or pathognomonic feature of ACC is the presence of high incidence of perineural invasion ${ }^{[6]}$; this accounts for an increased likelihood of positive microscopic surgical margins at the time of tracheal resection. The level of invasion that is seen microscopically is nearly always greater than what is grossly observed and hence complete resection may be quite difficult to achieve in most cases ${ }^{[7]}$.

Regional nodal metastasis has been reported to be around $10 \%$ and remote metastasis have very rarely been reported in the lungs, bones and brain ${ }^{[3]}$.

In contrast to squamous cell carcinomas (SCC) that occur in mainly in men $(90 \% \mathrm{v} / \mathrm{s} 10 \%)$, primary tracheal ACCs are found in men and women with almost equal frequency ${ }^{[4]}$. These tumours occur commonly occur between 45 to 60 years ${ }^{[4]}$; the mean age being 57.3 years ${ }^{[3]}$. These are usually not associated with smoking ${ }^{[9]}$. Our patient was a female in her fifth decade of life. Tracheal tumours typically present with signs or symptoms of upper airway obstruction such as cough, dyspnea, hoarseness, wheezing, stridor, dysphonia or hemoptysis ${ }^{[6]}$. Few patients can present with hoarseness and weight loss ${ }^{[4]}$. Symptoms are often present for some time and such patients would have been treated for asthma or chronic bronchitis prior to diagnosis ${ }^{[6]}$. The mean interval between symptom and diagnosis is 12 months ${ }^{[3]}$. Our patient presented with symptoms of hoarseness of voice and occasional dyspnea which was initially attributed to the thyroid enlargement (because of tumour infiltration) suggesting primary thyroid cancer with tracheal involvement.

Table. Comparison of adenoid cystic carcinoma with papillary thyroid carcinoma ${ }^{[5]}$

\begin{tabular}{lll}
\hline Items & ACC & PTC \\
\hline Cellularity & High & High \\
Pattern & Cribriform or trabecular & Papillae in sheets \\
Cytoplasm & Scant, basaloid & Moderate, squamoid \\
Nucleus & Oval to angulated & Round with grooves \\
Inclusions & None & Intranuclear \\
Nucleoli & Small, indistinct & Distinct \\
Chromatin & Coarse & Powdery \\
Mitoses & Uncommon & Rare \\
Background & Hyaline globules & Ropy colloid; psammoma bodies \\
\hline
\end{tabular}

Although chest X-rays are the first imaging usually performed in symptomatic patients, they are often non-diagnostic. On lateral neck X-rays, the tracheal air column usually reveals the presence of tumour but they are not useful in treatment planning. A three-dimensional (helical) CT-scan of the central airways provide precise anatomical information for endobronchial and surgical procedures ${ }^{[3]}$. CT scan in our case revealed a thyroid swelling with intra luminal extension of the tumour into the trachea.

FNAC can often be misleading in cases where tumour invades the thyroid gland as in our case. It is thought that an accurate diagnosis is difficult due to the FNA needle passing through the thyroid before reaching the trachea or a tumor that abuts both structures on imaging ${ }^{[10]}$. 
Although ACC of trachea is a low grade malignancy, more than $50 \%$ of patients eventually have haematogenous metastases. Pulmonary metastases are most common and can remain asymptomatic for many years ${ }^{[2]}$. Metastases to the brain, bone, liver, kidney, skin, abdomen and heart have also been reported ${ }^{[4]}$. Local recurrence is also common and occurs, on an average, 51 months after treatment. Lymphatic spread is however uncommon in patients with tracheal $\mathrm{ACCs}^{[4]}$.

Surgical resection is the treatment of choice for tracheal malignancies ${ }^{[6]}$. Tracheal resection and end-to-end tension free anastomosis are the primary form of therapy for ACC of the trachea ${ }^{[3]}$. Gaissert et al. ${ }^{[1]}$ suggested that resection of the trachea or carina in ACC was associated with long-term survival superior to palliative therapy particularly for cases with complete resection and negative airway margin. Complications of surgery include anastomotic dehiscence, tracheoesophageal fistula, pharyngeal or esophageal leaks, wound dehiscence, vocal cord palsy, temporary tracheostomy, dysphagia, ileus and pneumonia ${ }^{[4]}$. Our patient developed a small dehiscence of the anastomosis on the 4 th post-operative day with ensuing subcutaneous emphysema which was managed conservatively with oxygen supplementation and positive pressure breathing devices.

The role of post-operative adjuvant radiotherapy is not clear cut ${ }^{[4]}$. Radiotherapy is usually recommended for incomplete resections or positive margins where it might provide additional local disease control ${ }^{[4,6]}$. The recommended dose is between 54 and $60 \mathrm{~Gy}{ }^{[9]}$. For unresectable cases, external beam radiation has been used. Results of definitive radiotherapy for local control are between $20 \%$ and $70 \%$, with an improvement in outcome when tumour dose more than 60 Gy is utilized. Other modalities less commonly used are neutron beam radiotherapy and high dose rate endobronchial brachytherapy. These have been associated with a risk of damage to the bronchi resulting in fistulae or airway narrowing ${ }^{[6]}$. Chemotherapy does not appear to have a role in the treatment of ACC of the trachea ${ }^{[4]}$.

Laser therapy in tracheal tumours has been used as a palliative measure or that used after failure of other treatment modalities; most commonly used laser is the Nd-YAG laser. This technique may increase the survival when combined with other therapies. Debulking of tumour can be achieved with good precision; however it does not provide tissues for confirmation of resection and can leave tumour tissues behind. In combination with external beam radiation, it is useful in patients who refuse or those who are not candidates for surgery. It is also used as initial modality of treatment to completely clear the lumen in patients with stridor ${ }^{[9]}$.

Target therapy using imatinib has been recently studied in 16 patients with ACC of the salivary glands; however, studies have shown no benefit even in c-kit positive tumors ${ }^{[12]}$. In our case, the tumour was focally positive for c-kit mutation which can be used for treatment in metastatic setting.

The 5-year survival of tracheal ACC ranges from $66 \%$ to $100 \%$ and the 10 -year survival from $51 \%$ to $62 \%$ for patients regardless of the treatment ${ }^{[4]}$. Late recurrences can also occur after definitive therapy emphasizing the significance of prolonged surveillance in all treated patients ${ }^{[12]}$.

\section{Conclusion}

Diagnosing tracheal Adenoid cystic carcinomas can be a challenge as they are uncommon. Symptoms specific to the tumor mass are very rare and even so as thyromegaly are very rare. In the evaluation of a thyroid mass with tracheal involvement, ACC of trachea should be considered in the differential diagnosis with confirmation by bronchoscopy and biopsy along with immunohistochemistry. Rapid initial diagnosis and surgical treatment remains the mainstay of treatment. Adjuvant radiation therapy is used to achieve locoregional control. C-kit positivity has proven beneficial in metastatic disease. Long term follow up is mandatory. 


\section{References}

[1] Gopalakrishnan A, Unnikrishnan, Menon UV. Thyroid disorders in India: An epidemiological perspective. Indian J Endocrinol Metab. 2011; 15(Supp12): S78-S81. PMid: 21966658. http://dx.doi.org/10.4103/2230-8210.83329

[2] Ante M, Marija A, Mažuranić I. Extensive Tracheal Adenoid Cystic Carcinoma - Case Report J Clin Case Rep. $2012 ; 2: 224$.

[3] Bagheri R, Masum HSF, Majdi NMA, Rahim MB. Adenoid Cystic Carcinoma of the Trachea. Tanaffos. 2008; 7(4): $49-54$.

[4] Yang PY, Liu MS, Chen CH, Lin CM, Tsao TCY. Adenoid Cystic Carcinoma of the Trachea: a Report of Seven Cases and Literature Review. Chang Gung Med J. 2005; 28: 357-63. PMid: 16086551.

[5] Idowu MO, Reiter ER., Powers CN. Adenoid Cystic Carcinoma: A Pitfall in Aspiration Cytology of the Thyroid. Am J Clin Pathol. 2004; 121: 551-556. PMid: 15080307. http://dx.doi.org/10.1309/NKED4TJXUENR21M2

[6] Millar LPB, Stripp D, Cooper J, Both S, James P, Rengan R. Definitive Radiotherapy for Unresected Adenoid Cystic Carcinoma of the Trachea. Chest. 2012; 141(5): 1323-1326. PMid: 22553266. http://dx.doi.org/10.1378/chest.11-0925

[7] Vigg A, Mantri S, Vigg A, Vigg A. Adenoid Cystic Carcinoma of Trachea. Indian J Chest Dis Allied Sci. 2004; 46: 287-289. PMid: 15515830 .

[8] Nuwal P, Dixit R, Singhal AK. Primary adenoid cystic carcinoma of trachea presenting as midline neck swelling and mimicking thyroid tumor: A case report and review of literature. Lung India. 2010; 27: 167-9. PMid: 20931039. http://dx.doi.org/10.4103/0970-2113.68330

[9] Lay G, Amichetti M, Dessi M, Orru S, Versace R. Adenoid Cystic Carcinoma (ACC) of the Tracheo-Bronchial Tree Treated with Laser Therapy and Irradiation: Report of Two Cases The Open Lung Cancer Journal. 2009; 2: 31-34. http://dx.doi.org/10.2174/1876819900902010031

[10] Scherl S, Alon EE, Karle WE, Clain JB, Khorsandi A, Urken ML. Thyroid. 2013; 23(1): 79-83. PMid: 23072609. http://dx.doi.org/10.1089/thy.2012.0192

[11] Gaissert HA, Grillo HC, Shadmehr MB, et al. Long-term survival after resection of primary adenoid cystic and squamous cell carcinoma of the trachea and carina. Ann Thorac Surg. 2004; 78: 1889-96. PMid: 15560996. http://dx.doi.org/10.1016/j.athoracsur.2004.05.064

[12] Wang X, Zhao J, Fang X, Xu N, Zhang X. Adenoid Cystic Carcinoma to Low-Differentiation Carcinoma, Squamous Cell Carcinoma: A Rare Evolution of a Tracheal Malignancy Within 27 Years. Journal of clinical oncology diagnosis in oncology. 2011; 29(17). 